\title{
PRODUÇÃO DE CARVÃo ATIVADO A PARTIR DE CASCA DE ARROZ ${ }^{1}$
}

PRODUCTION OF CHARCOAL ACTIVATED FROM RICE HUSK

\author{
Amanda da Silva Reis \\ Niléia Cristina da Silva \\ Universidade Federal do Tocantins- UFT
}

Ubaldo Martins Neves

Universidade Federal de São Carlos

\begin{abstract}
RESUMO
A casca de arroz, um dos mais abundantes rejeitos agrícolas em nosso país, é um material fibroso com alto teor de sílica $\left(\mathrm{SiO}_{2}\right)$. O uso da casca de arroz como matéria prima para produção de carvão ativado é um projeto novo e o seu uso se deve à adequação de suas características básicas (não-grafitizável, alto teor de carbono fixo) para esta finalidade.

Este trabalho teve como objetivo a produção de carvão ativado a partir da casca de arroz. Após ser devidamente tratada a casca de arroz foi carbonizada a $600{ }^{\circ} \mathrm{C}$ por 40 minutos em uma mufla. $\mathrm{O}$ agente ativador utilizado na ativação química do material foi o Ácido Fosfórico $\left(\mathrm{H}_{3} \mathrm{PO}_{4}\right)$ concentrado $(85 \%)$.

Os testes realizados foram: $\mathrm{pH}$ de carga zero, métodos cromatográficos (adsorção do agrotóxicos), teste de CA como adsorvente de pesticidas, Análise de FTIR, Microscopia eletrônica de varredura MEV. Pela determinação do $\mathrm{pHpcz}$, o ponto de intersecção da curva está em 6.4, ou seja, mostra que o carvão ativado de casca de arroz é levemente ácido. O método cromatográfico (adsorção usando HPLC) mostrou que o carvão ativado de casca de arroz teve maior eficiência na remoção do pesticida Paration Metil da solução $(83,78 \%)$, que na remoção do pesticida $2,4 \mathrm{D}(21,61 \%)$.
\end{abstract}

Palavras chave: Carvão ativado; Casca de arroz; Paration metil; Ácido fosfórico $\left(\mathrm{H}_{3} \mathrm{PO}_{4}\right)$.

\section{ABSTRACT}

The rice husk, one of the most abundant agricultural wastes in our country, is a fibrous material with a high silica content $\left(\mathrm{SiO}_{2}\right)$. The use of rice husk as raw material for activated carbon production is a new project and its use is due to the adequacy of its basic characteristics (non-grafitizável, high fixed carbon content) for this purpose.

This study aimed to make charcoal activated from the rice husk. After rice husk being properly treated was charred to 600th $\mathrm{C}$ for 40 minutes in a muffle furnace. The activating agent used in the chemical activation of the material was Phosphoric Acid $\left(\mathrm{H}_{3} \mathrm{PO}_{4}\right)$ concentrate $(85 \%)$.

The tests were: $\mathrm{pH}$ of zero charge, chromatographic methods (pesticides adsorption), AC test as adsorbent pesticides, FTIR analysis, scanning electron microscopy SEM. For determining the $\mathrm{pHpcz}$, the point of intersection of the curve is at 6.4, that is, shows that the activated carbon from rice husk is slightly acidic. The chromatographic method (adsorption using HPLC) showed that the activated carbon rice hull was more efficient removal of methyl parathion pesticidal solution (83.78\%), which in the removal of 2,4 pesticide $\mathrm{D}(21.61 \%)$.

Keywords: Activated carbon; Rice husk; Methyl parathion; Phosphoric acid (H3PO4).

\footnotetext{
${ }^{1}$ Trabalho premiado no $10^{\circ}$ Seminário de Iniciação Científica da Universidade Federal do Tocantins, realizado de 24 a 28 de novembro de 2014, em Palmas-TO.
} 
Recebido em 10/09/2015. Aceito em 24/09/2015. Publicado em 03/12/2015.

\section{INTRODUÇÃO}

A casca de arroz, um dos mais abundantes rejeitos agrícolas em nosso país, é um material fibroso com alto teor de sílica $\left(\mathrm{SiO}_{2}\right)$. Seus principais componentes são celulose (juntamente com outros carboidratos), lignina e resíduos inorgânicos (Lee \& Cutler, 1975; James \& Rao, 1986). Esforços para tentar aproveitar a casca de arroz são dificultados pela sua grande dureza, baixo teor nutritivo, grande resistência à degradação e grande teor de cinzas (tipicamente de 15 a $20 \%$ em massa, dos quais cerca de 95\% correspondem a sílica) (Della et al., 2001, Sun \& Gong, 2001).

Na safra 2008/09 o Brasil produziu 12,638 milhões de toneladas de arroz em casca. A região sul é a maior produtora, destacando o estado do Rio Grande do Sul (RS) como o grande produtor nacional. A safra de 2008/09 do RS chegou a 8,047 milhões de toneladas, o que correspondeu a 63\% da produção total (Cogo, 2009). Dessa maneira, são geradas, anualmente, cerca de 2,74 milhões de toneladas de casca de arroz no país, que podem ser aproveitadas para diversas funções, dentre elas a produção de carvão ativado.

Como a remoção dos poluentes nos tratamentos de águas convencionais é muitas vezes incompleta, estas contêm frequentemente muitas substâncias químicas dissolvidas, as quais podem afetar os ecossistemas e o abastecimento das águas potáveis (RODRIGUEZ-MOZAZ et all, 2004).

A determinação do tratamento de água contaminada depende de fatores como a natureza dos poluentes, sua concentração, volume a tratar e toxicidade. A adsorção com carvão ativado é a tecnologia mais utilizada na purificação de águas contaminadas por pesticidas e outros compostos químicos perigosos. O carvão ativado é um material carbonoso poroso que apresenta uma forma microcristalina, não grafítica, que sofreu um processamento para aumentar a porosidade interna. Uma vez ativado o carvão apresenta uma porosidade interna comparável a uma rede de tuneis que se bifurcam em canais menores e assim sucessivamente. Esta porosidade diferenciada e classificada segundo o tamanho dos poros, divididas em macro, meso e microporosidade (VALENCIA, 2007).

O carvão ativado elimina substâncias de concentração, composição e tamanhos variáveis (REINOSO, 2004). Este material possui aplicações múltiplas e tem sido empregados 
na indústria de alimentos, bebidas, farmacêutica, química, etc. No tratamento de água para consumo humano, o carvão ativado tem a capacidade de eliminar o sabor e o odor da água, eliminar contaminantes como metais pesados, gases tóxicos, pesticidas, reduzir a matéria orgânica natural, limitando assim ao máximo a formação de subprodutos de desinfecção e/ ou oxidação, como trihalometanos (PHAN et al., 2006; AKSU et al., 2008). A principal característica do C.A e a de conter uma alta superfície específica, a qual depende da sua microporosidade elevada (L. Li et al., 2002). A estrutura química do carvão ativado (Figura 1).

Figura 1: Estrutura química básica do carvão ativado

Figure 1: Basic chemistry of activated carbon structure

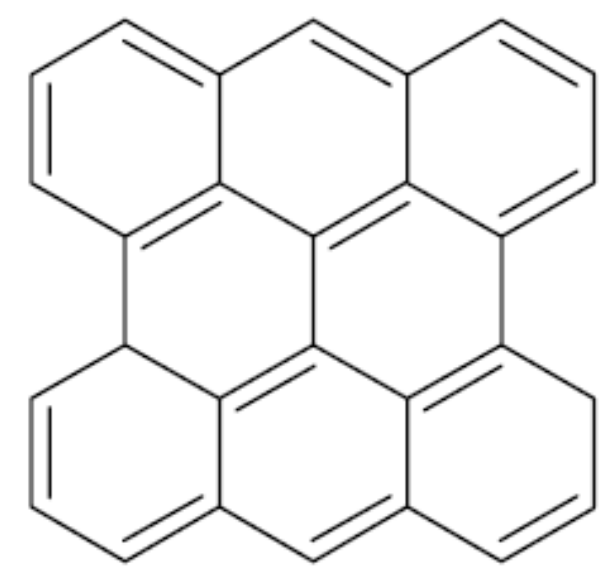

Fonte: Barros (2008).

A capacidade de adsorção do carvão ativado e determinada, entre outros fatores, por sua area superficial especifica, por sua estrutura porosa interna e pela presença de grupos funcionais sobre a superfície (SOLEIMANI; KAGHAZCHI, 2008).

O processo da adsorção que utiliza carvão ativado comercial é muito eficaz para a remoção de contaminantes de águas residuárias, entretanto seu custo elevado promove a busca de alternativas por adsorventes de baixo custo.

O uso da casca de arroz como matéria prima para produção de carvão ativado é um projeto novo e o seu uso se deve à adequação de suas características básicas (não-grafitizável, alto teor de carbono fixo) para esta finalidade.

Este trabalho teve como objetivo a produção de carvão ativado a partir da casca de arroz. O agente ativador utilizado na ativação química do material foi o Ácido Fosfórico $\left(\mathrm{H}_{3} \mathrm{PO}_{4}\right)$ concentrado. 


\section{MATERIAIS E MÉTODOS}

O fluxograma a seguir apresenta todas as etapas envolvidas no projeto, a preparação do material precursor, carbonização do material, ativação do material.

Figura 2: Fluxograma descritivo da metodologia utilizada no projeto.

Figure 2: Descriptive flow chart of the methodology used in the project.

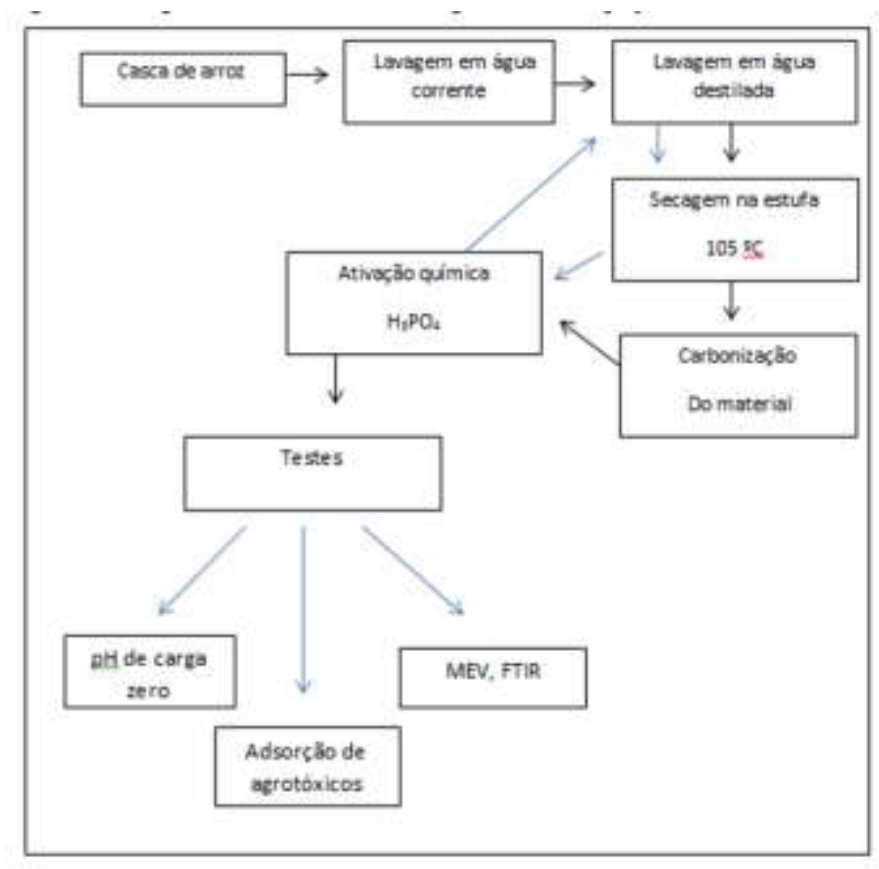

Fonte: Reis (2015).

\subsection{Preparo das amostras e carbonização do material}

A casca de arroz foi separada e lavada em água corrente para a retirada das impurezas externas. Em seguida a casca de arroz foi lavada por 50 minutos na água destilada em ebulição, logo depois o material foi lavado e filtrado em água destilada à temperatura ambiente $\left(25^{\circ} \mathrm{C}\right)$. Após a lavagem, o material foi colocado para secar na estufa à $105^{\circ} \mathrm{C}$ por 24 horas.

Tendo se passado às 24 horas o material foi encaminhado para a carbonização, onde foi dividido e colocado em quatro "cadinhos" previamente enumerados e pesados, para que depois fosse possível calcular qual foi a perda de massa de cada amostra.

O material foi colocado no forno (Mufla) para carbonizar a $600^{\circ} \mathrm{C}$ por 40 minutos, após a retirada do material da Mufla, o mesmo foi colocado no dessecador para esfriar por 24 horas. 


\subsubsection{Ativação do material}

O processo de ativação utilizado no carvão foi o processo químico, onde o agente ativador foi o Ácido Fosfórico $\left(\mathrm{H}_{3} \mathrm{PO}_{4}\right)$ concentrado $(85 \%)$.O material foi retirado dos cadinhos e colocado em um Becker de $500 \mathrm{ml}$, onde foi adicionado o ácido até que o material sobrenadasse. Logo depois o mesmo foi colocado na chapa agitadora, para ficar em agitação por 24 horas.

Decorridas as 24 horas de agitação o material foi lavado em agua destilada e filtrado até atingir o pH 5.8, em seguida foi colocado na estufa para secar por 24 horas à $100^{\circ} \mathrm{C}$. Ao término dessas 24 horas, o material foi macerado em um cadinho até ficar com a textura em fina.

Figura 3: Material já carbonizado Figure 3: Carbonized material

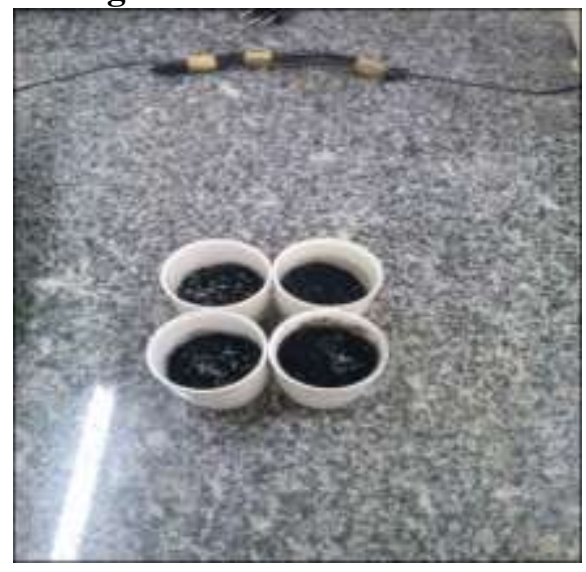

Fonte: própria autora
Figura 4: Material macerado Figure 4: Macerated Material

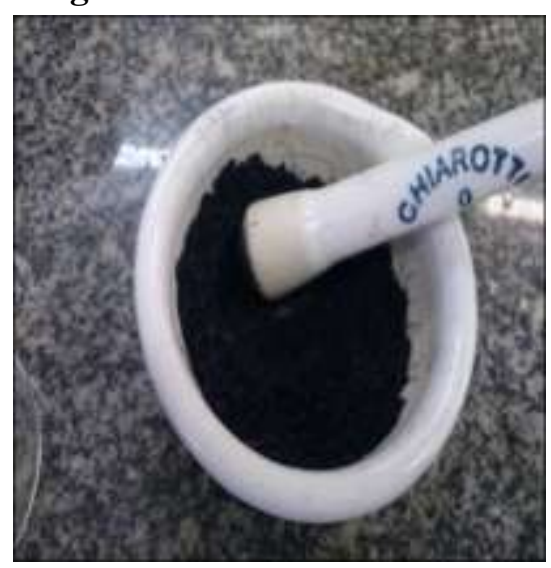

Fonte: própria autora

\subsection{Caracterização do CA}

\subsubsection{Determinação do $\mathrm{pH}$ de ponto de carga zero $\left(\mathrm{pH}_{p c z}\right)$}

O ponto de carga zero é definido como o $\mathrm{pH}$ em que a superfície do carvão possui carga neutra(AHMAD E KUMAR, 2010). A Metodologia empregada para sua determinação é denominada "experimento dos 11 pontos" (GUILARDUCE et all.,2006).

O procedimento consistiu em fazer a mistura de $0,1 \mathrm{~g}$ do CCA em $100 \mathrm{ml}$ de solução aquosa de $\mathrm{NaCl}(0,1 \mathrm{~mol} / \mathrm{L})$ sob diferentes condições de $\mathrm{pH}$ inicial $(1,2,3,4,5,6,8,9,10,11 \mathrm{e} 12)$ e medir o pH após 24 horas de equilíbrio. Fazendo-se o gráfico de $\mathrm{pH}$ inicial versus pH final, o $\mathrm{pH}_{\mathrm{pcz}}$ corresponde a faixa onde o $\mathrm{pH}$ final se mantém constante independente do $\mathrm{pH}$ inicial, ou seja, a superfície comporta-se como um tampão(SHARMA et all., 2009). 


\subsubsection{Espectroscopia de Infra Vermelho por Transformada de Fourier Acoplada à técnica de Refletância Total Atenuada (FTIR-ATR)}

A analise de FTIR-ATR foi realizado em colaboração com o professor Edemir Feliciano Garcia na Universidade Estadual de São Paulo (UNESP), Campus de Ilha Solteira.

A caracterização química da superfície dos adsorventes, antes e depois da adsorção, foi realizada por FTIR. Existem diferentes metodologias de estudo dos grupos funcionais disponíveis em materiais porosos, entretanto a espectroscopia no infravermelho tem se mostrado eficiente nessa avaliação (SEREDYCH, 2008).

O teste foi feito em um espectrofotômetro (PerkinElmer UATR Two), Figura 5. Os espectros foram obtidos com 20 varreduras, na região de 4000 a $400 \mathrm{~cm}^{-1}$, e foi aplicado um torque de $79 \mathrm{~N}$ com o braço articulado.

Figura 5: Espectrômetro PerkinElmer UATR Two.

Figure 5: PerkinElmer Spectrometer UATR Two

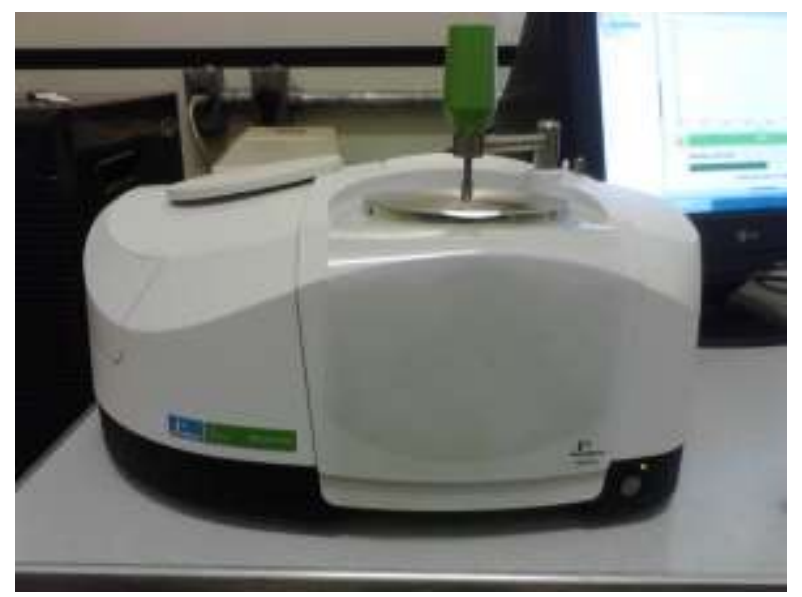

Fonte: Garcia, 2014

\subsubsection{Microscopia eletrônica de varredura}

As morfologias superficiais dos materiais foram analisadas por microscopia eletrônica de varredura (MEV). A avaliação da morfologia dos adsorventes foi realizada por meio de micrografias obtidas por um microscópio eletrônico EVO LS15 da marca Carl Zeiss, disponível no Departamento de Química e Física da UNESP-FEIS. 
Essa análise de microscopia eletrônica de varredura nas amostras após a obtenção do adsorvente, com o intuito de avaliar a superfície do material observar as diferenças morfológicas decorridas do processo de carbonização.

\subsection{Métodos Cromatográficos (adsorção do agrotóxico)}

Este teste foi realizado em colaboração com o professor Edemir Feliciano Garcia na Universidade Estadual de São Paulo (UNESP), Campus de Ilha Solteira.

A determinação da adsorção dos pesticidas e a quantificação da capacidade de remoção foram feitas por cromatografia Liquida de alta Performance (ou HPLC), a figura Figura 6 ilustra uma visão geral do aparelho utilizado.

Figura 6: Visão do aparelho HPLC utilizado neste trabalho.

Figure 6: HPLC machine vision used in this work.

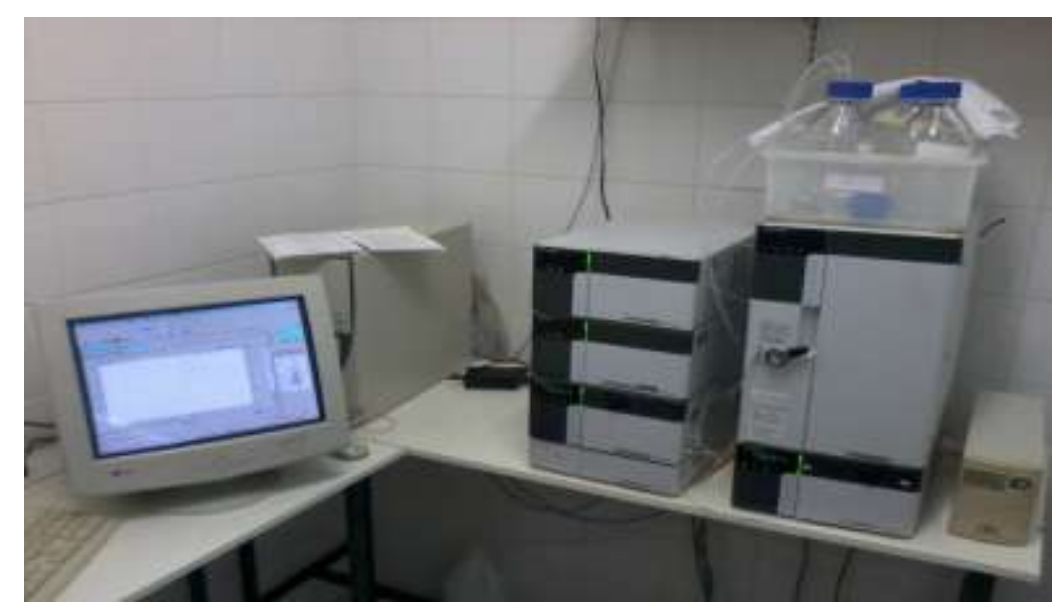

Fonte: Garcia, 2014

As análises quantitativas dos pesticidas alvos foram realizadas mediante as seguintes condições cromatográficas: cromatógrafo liquido de alta performance da marca Shimizu, equipado com detector "Photodiode Array" (SPD-M20A), duas bombas de alta pressão (LC20AT e LC 20AD), em coluna C-18 (modelo Shim-pack), com 4,6 x 250 mm e diâmetro de partícula de $5 \mu \mathrm{m}$, empregando o software LCsolution. As condições de adsorção que foram usadas no processo são descritas na Tabela 1. 
Tabela 1: Condições de Adsorção

Table 1: Conditions of Adsorption

\begin{tabular}{l|l|l}
\hline \multicolumn{3}{c}{ Condições de adsorção } \\
\hline Massa & $0,100 \mathrm{~g}$ & \\
\hline ol & 0,0501 & \\
\hline Agit & $175 \mathrm{rpm}$ & \\
\hline Temp & Ambiente & \\
\hline Conc Inic & $2,4 \mathrm{D}$ & $0,253914 \mathrm{mmol} / \mathrm{L}$ \\
\hline & Paration metil & $0,51564 \mathrm{mmol} / \mathrm{L}$ \\
\hline
\end{tabular}

Fonte: Própria autora

\subsection{Teste de CA como adsorvente de pesticidas}

Foram realizados ensaios para avaliar a porcentagem de remoção de pesticida da agua, através do processo de batelada, um volume de $0,05 \mathrm{~L}$ de solução de pesticidas a uma concentração de $0,253914 \mathrm{~mm} / \mathrm{moL}$ para $2,4 \mathrm{D}$ e $0,51564 \mathrm{~mm} / \mathrm{moL}$ para paration metil, foi mantido em contato com $100 \mathrm{mg}$ de adsorvente por $120 \mathrm{~min}$, a uma velocidade de agitação de aproximadamente 170 rpm, em uma mesa de agitação orbital.

Tabela 2: Condições do Teste de Adsorção

Table 2: Adsorption Test Condition

\begin{tabular}{lcc}
\hline \multicolumn{1}{c}{ Parâmetros } & \multicolumn{2}{c}{ Pesticida } \\
\cline { 2 - 3 } & $2,4-\mathrm{D}$ & PM \\
\hline $\begin{array}{l}\text { Concentração inicial } \\
\left(\text { mmol.L }{ }^{-1)}\right.\end{array}$ & 0,25 & 0,51 \\
\hline Massa do adsorvente $(\mathrm{mg})$ & 100 & 100 \\
\hline Vol. de Solução (mL) & 50 & 50 \\
\hline Agitação (rpm) & 170 & 170 \\
\hline Temperatura ${ }^{\circ} \mathrm{C}$ & $25 \pm 2$ & $25 \pm 2$ \\
\hline Tempo de agitação (min) & 120 & 120 \\
\hline
\end{tabular}

Fonte: Própria autora

Depois de decorrido o tempo de contato, a amostra foi imediatamente filtrada através de membrana de $0,45 \mu \mathrm{m}$, a fração filtrada era então submetida à análise, seguindo o método descrito no item 3.7, desta forma, verificando-se a percentagem de remoção do pesticidas, (através da Equação 1) no determinado tempo até que a concentração atingisse o equilíbrio. 


$$
\% \text { removido }=\frac{\left(\mathrm{C}_{0}-\mathrm{C}_{\mathrm{e}}\right)}{\mathrm{C}_{0}} \times 100
$$

Sendo $\mathrm{C}_{0}$ a concentração inicial da solução em mg/L e $\mathrm{C}_{\mathrm{e}}$ a concentração residual de pesticidas após o tempo de contato com o adsorvente também em mg/L. O procedimento foi realizado em triplicata.

\section{RESULTADOS E DISCUSSÕES}

\subsection{Caracterização do CA}

\subsection{1 $\mathrm{pH}$ de ponto de carga zero $\left(\mathrm{pH}_{\mathrm{pcz}}\right)$}

A determinação do $\mathrm{pH}_{\mathrm{pcz}}$ foi realizada no intuito de investigar a carga da superfície do CA usado no experimento. $\mathrm{O}$ gráfico foi feito plotando o $\mathrm{pH}$ final versus o $\mathrm{pH}$ inicial, onde o ponto de intersecção da curva está em 6.4, como pode ser visto na figura 7.Pela determinação do $\mathrm{pH}_{\mathrm{pcz}}$ observa-se que o carvão ativado é levemente ácido.

Figura 7: Determinação do $\mathrm{pH}_{\mathrm{pcz}}$ do carvão ativado de casca de arroz

Figure 7: $\mathrm{pH}$ determination $\mathrm{PCZ}$ of activated charcoal rice husk

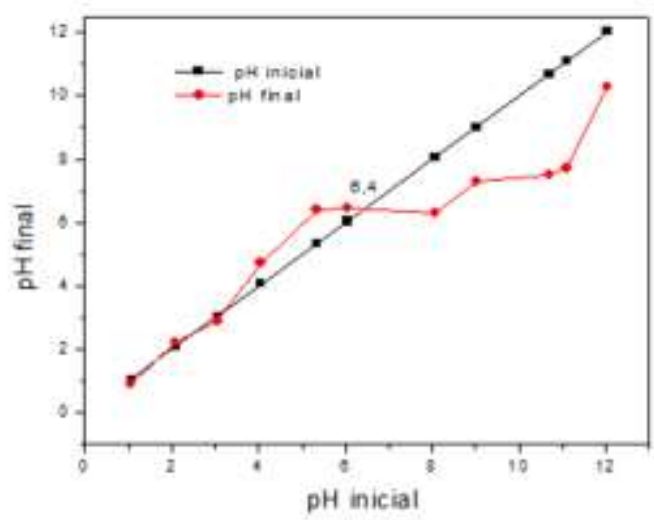

Fonte: Silva, 2014
Figura 8: Carga da Superfície em Função do PHpcz.

Figure 8: Surface load in PHpcz function.

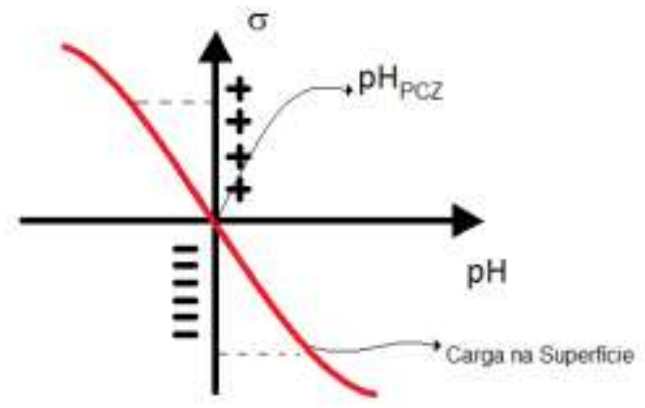

Fonte: Própria Autora

MORENO-CASTILLA (2004) mostra que os grupos funcionais que determinam as características ácidas e básicas, nas superfícies desses materiais podem ser separados em dois 
grupos: A) ácidos - carboxílicos, lactonas, fenólicos, cetônicos, quinonas álcoois e éteres e B) Básicos - pironas e cromenos, além dos grupos cetona e éteres, conforme pode ser visto na Figura 9. O grupo A torna o material mais hidrofílico e ácido diminuindo o valor do $\mathrm{pH}$ e do $\mathrm{pH}_{\mathrm{PCZ}}$, por outro lado os complexos superficiais de oxigênio formados grupo B atuam como sítios básicos, se comportando como base de Lewis e recebe prótons em solução.

Figura 9: Grupos funcionais encontrados na superfície de materiais carbonosos. Figure 9: Functional groups found on the surface of carbonaceous materials.

Grupo A

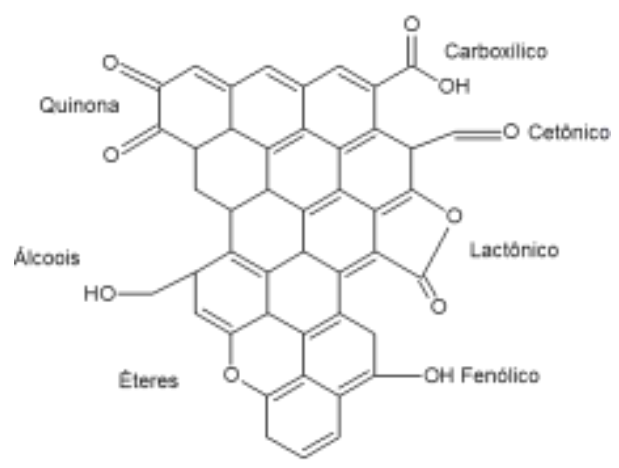

Grupo B

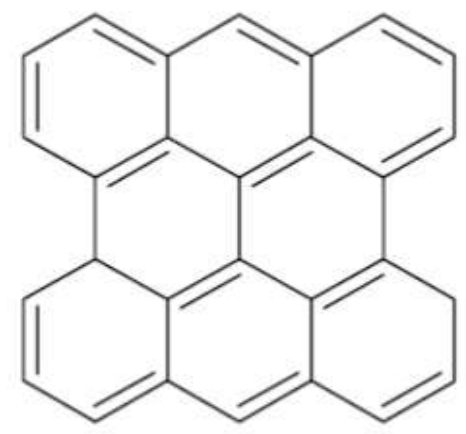

Fonte: Adaptado de MORENO-CASTILLA (2004)

\subsubsection{Espectroscopia de Infravermelho por Transformada de Fourier}

Os resultados de FTIR (figura 10) apresentaram aumento na intensidade entre 3250 e $3500 \mathrm{~cm}^{-1}$, caracteriza-se uma banda de absorção típica de $(\mathrm{OH})$, que normalmente também está associada à presença de hidrogênio. Essa absorção pode ser atribuída à presença predominantemente de grupos $\mathrm{OH}$ de fenóis, pois em geral, a presença significativa de $(\mathrm{OH})$ de grupos carboxílicos em CA se caracteriza por forte absorção, que se estende até $2500 \mathrm{~cm}$ (GUILARDUCI et al, 2006).

$\mathrm{O}$ aumento de intensidade entre 1500 e $1750 \mathrm{~cm}^{-1}$ evidenciam a presença de grupos de carbonila $\mathrm{C}=\mathrm{O}$, existentes em ácidos carboxílicos, cetonas e na própria celulose. A presença da banda em aproximadamente $1650 \mathrm{~cm}^{-1}$ pode ser atribuída a vibrações de anéis aromáticos típicos em materiais carbonáceos

O pico entre 1000 e $1250 \mathrm{~cm}^{-1}$ pode ser atribuído ao estiramento da ligação C-O correspondente as vibrações dos grupos fenólicos. Um pico de menor intensidade próximo a $600 \mathrm{~cm}^{-1}$ comprova a presença de éter e lactona (GARG et al.,2008 \& LIMA et al., 2008) 
Figura 10: Gráfico da espectroscopia de infravermelho por transformada de fourier.

Figure 10: Figure Spectroscopy Infrared Fourier transform.

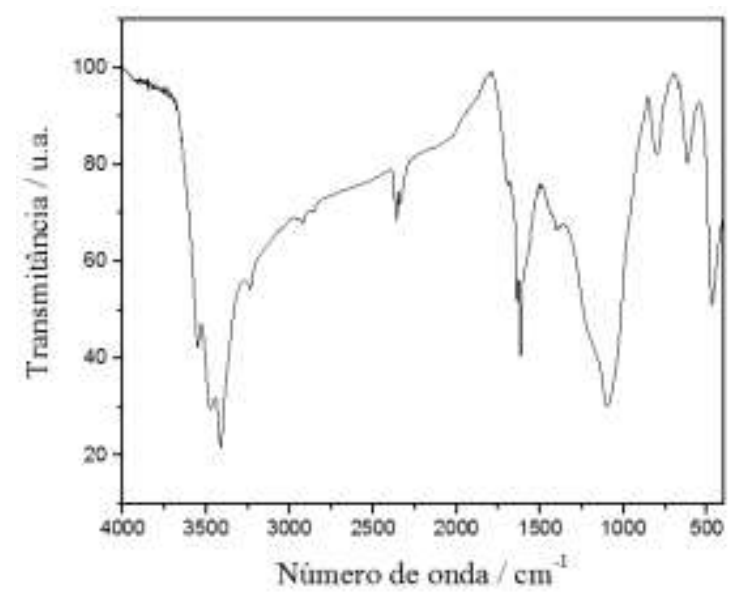

Fonte: Silva, 2015

\subsubsection{Microscopia eletrônica de varredura}

A morfologia do CA de casca de arroz foi estudada por microscopia eletrônica de varredura (MEV), MEV é uma técnica amplamente utilizada para estudar a morfologia da superfície características do adsorvente. No presente estudo, a MEV é utilizada para avaliar a morfologia do CA.

A imagem de MEV do CA é apresentada na Figura 11. A Micrografia revela uma superfície heterogênea altamente porosa.

Figura 11- Micrografia obtida por MEV do CA de casca de arroz.

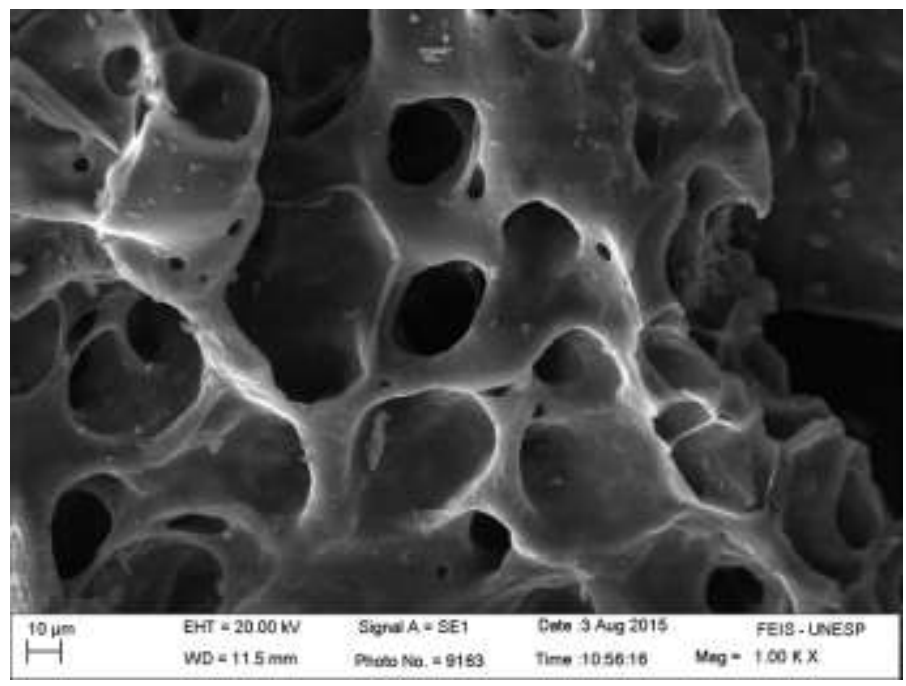

Fonte: Silva, 2015 


\subsection{Adsorção de pesticidas usando CA}

Como pode ser observado na figura 12, o carvão teve maior eficiência na remoção do pesticida Paration Metil da solução (83,78\%), que na remoção do pesticida 2,4 D $(21,61 \%)$. Assim, considerando a solubilidade em água (900mg.L $\left.{ }^{-1}\right)$, baixo valor de $\operatorname{KOW}(0,83)$ e polaridade (polar) do 2,4-D, sua adsorção em carvão ativado tende a ser reduzida, uma vez que o CA é um adsorvente hidrofóbico, apolar (ROZÁRIO, 2012).

Figura 12 : Adsorção por HPLC ( 2,4 D e Paration Metil)

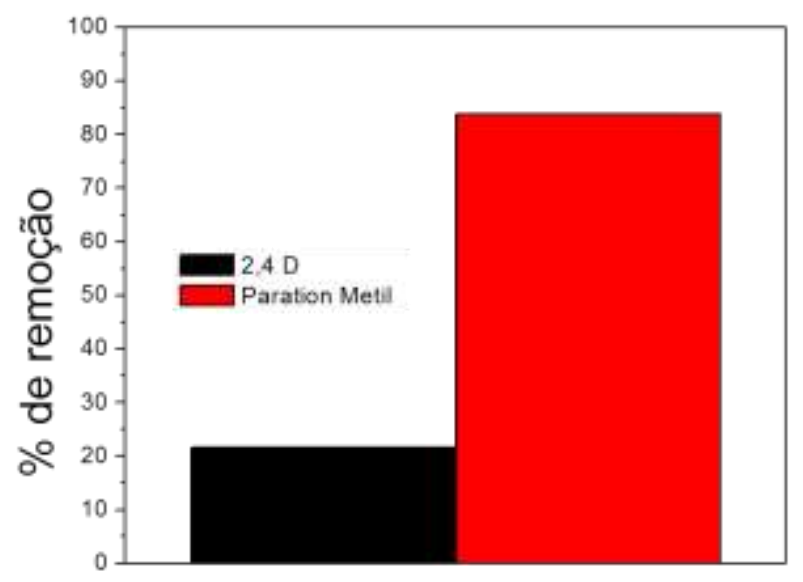

Fonte: Silva, 2014.

\section{REFERÊNCIAS}

AYGÜN, A.; YENISOY-KARAKAS, S.; DUMAN, I. Production of granular activated carbon from fruit stones and nutshells and evaluation of their physical, chemical and adsorption properties. Microporous and Mesoporous Materials, Amsterdam, v. 66, n. 2-3, p. 189-195, 2003.

AHMAD, R.; KUMAR, R. Conducting Polyaniline/Iron Oxide Composite: A Novel Adsorbent for the Removal of Amido Black 10B. Journal of Chemical and Engineering Data, Washington, DC, v. 55, n. 9, p. 3489-3493, 2010.

CLARK, H. L. M. Remoção de fenilalanina por Adsorvente produzido a partir da torta Prensada de grãos defeituosos de café. 115 f. Dissertação (Mestrado em Ciência de Alimentos) - Universidade Federal de Minas Gerais, Belo Horizonte, 2010.

COELHO, T.L. Estudos de adsorção de Cobre (II) em microesferas de quitosana com epicloridina e impregnadas com heparina, Florianópolis, Programa de Pós-Graduação em Engenharia Química, UFSC, 2006. Dissertação de Mestrado.

CRISPIM, S. C. L. Remoção de cálcio (II) em solução aquosa por meio de carvões ativados submetidos a tratamentos superficiais. 136 f. Tese (Doutorado em Química Analítica) Universidade Federal da Paraíba, João Pessoa, 2009.

DELLA,V.P. et all ., CARACTERIZAÇÃO DE CINZA DE CASCA DE ARROZ PARA USO COMO MATÉRIA-PRIMA NA FABRICAÇÃO DE REFRATÁRIOS DE SÍLICA. Departamento 
de Engenharia Mecânica/Departamento de Ciência e Engenharia de Materiais, Quim. Nova, Vol. 24, No. 6, 778-782, 2001. Universidade Federal de Santa Catarina, Florianópolis - SC. ABDEL-NASSER, EL-HENDAWY, A., "Surface and adsorptive properties of carbons prepared from biomass”. Applied Surface Science 252 (2005) 287-295.

FERNANDES, R. Adsorventes alternativos para remoção de fenol em solução aquosa. $91 \mathrm{f}$. Dissertação (Mestrado em Engenharia Química) - Universidade Federal de Santa Catarina, Florianópolis, 2005.

FERNANDES, F. L. Carvão de endocarpo de coco da baía ativado quimicamente com ZNCL2 e fisicamente com vapor d'água: produção, caracterização, modificações químicas e aplicação na adsorção de íon cloreto. 116 f. Tese (Doutorado em Química Analítica) Universidade Federal da Paraíba, João Pessoa, 2008.

Garg, U.; Kaur, M. P.; Jawa, G. K.; Sud, D.;Garg, V. K. Removal of cadmium (II) from aqueous solutions by adsorption on agricultural waste biomass. Journal of Hazardous Materials 2008, 154, 1149.

GUO, Y.; ROCKSTRAW, D. A., Activated carbons prepared from rice hull by one-step phosphoric acid activation, Microporous and Mesoporous Materials, 100: 12, 2007.

Lima, E. C.; Royer, B.; Vaghetti, J. C. P.; Simon, N. M.; da Cunha, B. M.; Pavan, F. A.; Benvenutti, E. V.; Cataluña-Veses, R.; Airoldi, C. Application of Brazilian pine-fruit shell as biosorbent to removal of reactive red 194 textile dye from aqueous solution: kinetics and equilibrium study. Journal of Hazardous Materials 2008, 155, 536.

GUILARDUCI, V.V.S., MESQUITA, J.P., MARTELlI, P.B., GORGULHO, H.F., "Adsorção de fenol sobre carvão ativado em meio alcalino". Química Nova, 29, 1226-1232, 2006.

GOLIN, D. M. Remoção de chumbo de meios líquidos através de adsorção utilizando carvão ativado de origem vegetal e resíduos vegetais. $124 \mathrm{f}$. Dissertação (Mestrado em Engenharia Ambiental) - Universidade Federal do Paraná, Curitiba, 2007.

JUNIOR, M. A. S. Ativação química do carvão de casca de arroz utilizando NaOH. $79 \mathrm{f}$. Dissertação (Mestrado em Física) - Universidade Federal do Espírito Santo, Vitória, 2004. LEI LI, P. A. et al. Effects of activated carbon surface chemistry and pore structure on the adsorption of organic contaminants from aqueous solution. Carbon, Oxford, v. 40, p. 20852100, 2002.

LEYVA-RAMOS, R., FUENTES-RUBIO, L., GUERRERO-CORONADO, R. M. et al. "Adsorption of trivalent chromium from aqueous solutions onto actived carbon". Journal of Chemical Technology and Biotechnology 62, pp. 64-67. 1995. 73

LYUBCHIK, S. I., LYUBCHIK, A. I., GALUSHKO, O. L. et al. "Kinetics and thermodynamics of the Cr(III) adsorption on the activated carbon from co-mingled wastes". Colloids and Surfaces A: Physicochemical Engineering Aspects 242, pp. 151- 158. 2004.

MORENO-CASTILLA, C. Adsorption of organic molecules from aqueous solutions on carbon materials. Carbon 42 (2004) 83-94, v. 42, p. 83-94, 2004.

NAMASIVAYAM, C.; SANGEETHA, D., Aplication of coconut coir pith for the removal of sulfate and other anions from water, Desalination, 219: 1, 2008.

NAMASIVAYAM, C.; SANGEETHA, D., Removal and recovery of vanadium (V) by adsorption onto $\mathrm{ZnCl}_{2}$ activated carbon : Kinetics and isotherms, Adsorption, 12: 103, 2006.

NIEDERSBER, C. Ensaios de adsorção com carvão ativado produzido a partir da casca do tungue (Aleurites Fordii), resíduo do processo de produção de óleo. Dissertação de Mestrado em Tecnologia Ambiental. Outubro 2012.

PHAN, N. H. et al. Production of fibrous activated carbons from natural cellulose (jute, coconut) fibers for water treatment applications, Carbon. v. 44. p. 2569-2577, 2006. 
PEREIRA, E. I. Produção de carvão ativado a partir de diferentes precursores utilizando $\mathrm{FeCl}_{3}$ como agente ativante. $90 \mathrm{f}$. Dissertação (Mestrado em Agroquímica) - Universidade Federal de Lavras, Minas Gerais, 2010.

ROCHA, W. D. Carvão ativado a partir de resíduos agrícolas e suas aplicações na adsorção de íons metálicos. 126 f. Dissertação (Mestrado em Engenharia Mineral) - Universidade Federal de Ouro Preto, Minas Gerais, 2006.

RODRIGUEZ-MOZAZ, S.; ALDA, M. J. L.; BARCELÓ, D. 2004. Monitoring of estrogens, pesticides and bisphenol $A$ in natural waters and drinking water treatment plants by solidphase extraction-liquid chromatography-mass spectrometry. Journal of Chromatography A, 1045: 85-92.

RADHIKA, M.; PALANIVELU, K., Adsorptive removal of chlorophenols from aqueous solution by low cost adsorbent - Kinetics and isotherm analysis, Journal of Hazardous Materials, B138, 116, 2006

ROZÁRIO, A. Avaliação da remoção do ácido 2,4-diclorofenoxiacético (2,4-D) em águas através do uso de carvão granular (CAG) em pequenas colunas (escala experimental).Dissertação (Mestrado) - Universidade Federal do Espírito Santo, 2012.

SAI, P. M. S.; KRISHNAIAH, K. Development of the pore-size distribution in activated carbon produced from coconut shell char in a fluidized-bed reactor. Industrial \& Engineering Chemistry Research, Washington, v. 44, n. 1, p. 51- 60, Jan. 2005.

SILVA, N. C. D. Remoção de Antibióticos da Água Por Meio do Processo de Adsorção em Carvão Ativado. 2012, 100 f. Dissertação (Mestrado em Química de Materiais) Faculdade de Engenharia da Universidade Estadual Pau-lista - UNESP. Ilha Solteira. 2012.

SEREDYCH, M., HULICOVA-JURCAKOVA, D., LU, G.Q., BANDOSZ, T.J., Surface functional groups of carbons and the effects of their chemical character, density and accessibility to ions on electrochemical performance, Carbon, 46 (2008) 1475-1488.

SOLEIMANI, M., KAGHAZCHI, T., "Adsorption of gold ions from industrial wastewater using activated carbon derived from hard shell of apricot stones - An agricultural waste." 13 (2008) Bioresource Technology 5374-5383

STAVROPOUlOS, G. G.; ZABANIOTOU, A. A. Production and characterization of activated carbons from olive-seed waste residue. Microporous and Mesoporous Materials, v. 82, p. 79-85, 2005.

SILVA, J. Estudo da eficiência e aplicabilidade de carvão ativado resultante de pirólise de casca de arroz em barreiras reativas na remoção de contaminantes em solos. $204 \mathrm{f}$. Tese (Doutorado) - Universidade Federal do Rio Grande do Sul, Porto Alegre, 2009.

SOARES, W. L. Uso de agrotóxicos e seus impactos à saúde e ao meio ambiente: uma avaliação integrada entre a economia, a saúde, a ecologia e a agricultura. Tese (Doutora-do) - Escola Nacional de Saúde Pública Sérgio Arouca. Rio de Janeiro, 2010.

SCHNEIDER, E. L. Adsorção de compostos fenólicos sobre carvão ativado. Toledo: Centro de Engenharias e Ciências Exatas da UNIOESTE, 2008. 79 p. (Dissertação, Mestrado em Engenharia Química).

SHARMA, Y. C. et al. Removal of a cationic dye from wastewaters by adsorption on activated carbon developed from coconut coir. Energy \& Fuels, Washington, DC, v. 23, n. 6, p. 2983-2988, 2009.

SCHIMMEL, D. ; et al . Adsorption of turquoise blue qg reactive dye on commercial activated carbon in batch reactor: kinetic and equilibrium studies. Brazilian Journal of Chemical Engineering (Impresso) Icr, v. 27, p. 289-298, 2010.

TOCANTINS. SECRETARIA DA AGRICULTURA, DA PECUARA E DO DESENVOLVIMENTO AGRÁRIO. Projetos Agrícolas do Tocantins, 2012. Disponível em:< http://seagro.to.gov.br/>. Acesso em : 28 de ago. 2012. 
VALENCIA, C. A. V. Aplicação da adsorção em carvão ativado e outros materiais carbonosos no tratamento de águas contaminadas por pesticidas de uso agrícola. 2007. 115 f. Dissertação (Mestrado em Engenharia Metalúrgica) - Pontifícia Universidade Católica do Rio de Janeiro, Rio de Janeiro, 2007.

WERLANG, E. B. et al., Produção de carvão ativado a partir de resíduos vegetais. Revista Jovens Pesquisadores, Santa Cruz do Sul, v. 3, n. 1, p. 156-167, 2013.

YOUSSEF, A. M., El-NABARAWY Th. SAMRA, S. E. "Sorption properties of chemicallyactivated carbons 1. Sorption of cadmium(II) ions". Colloids and Surfaces A: Physicochemical Engineering Aspects 235, p. 153. 2004.

\section{Amanda da Silva Reis}

Aluna do Curso de Engenharia Florestal; Campus de Gurupi.

E-mail: areis176@gmail.com

Endereço: Curso de Agronomia da Universidade Federal do Tocantins (UFT), Rua Badejós, Lote 7, Chácaras 69/72, Zona Rural. Cx.postal 66. CEP: 77402-970 - Gurupi (TO)

\section{Niléia Cristina da Silva}

Possui graduação em Licenciatura em Química, especialização em Química Industrial pelo Centro Universitário de Votuporanga (2007), Mestrado e Doutorado em Ciências dos Materiais pela UNESP-Universidade Estadual Paulista, Campus de Ilha Solteira. Atualmente é Professora Assistente do Curso de Agronomia da Universidade Federal do Tocantins (UFT), Campus de Gurupi.

E-mail: nilacs@uft.edu.br

Endereço: Curso de Agronomia da Universidade Federal do Tocantins (UFT), Rua Badejós, Lote 7, Chácaras 69/72, Zona Rural. Cx.postal 66. CEP: 77402-970 - Gurupi (TO)

\section{Ubaldo Martins Neves}

É Bacharel, Mestre e Doutor em Física Aplicada pela Universidade de São Paulo tendo como tema de estudo na pós-graduação as propriedades ópticas de materiais. Atualmente é professor de Matemática e Física na Universidade Federal de São Carlos campus Lagoa do Sino.

E-mail: neves@mail.uft.edu.br

Endereço: Universidade Federal de São Carlos, Universidade Federal de São Carlos. Campus Lagoa do Sino Rodovia Lauri Simões de Barros, km 12 - SP-189- Bairro Araraçú 77402970 - Buri, SP - Brasil 